\title{
Peri-Operative Assessment of Critical Aortic Stenosis Prior to Non-cardiac Surgical Procedures
}

Molly Amin MD, Faraz Chaudhry MD, Anthony Sifonios MD, and Antonio Gonzalez-Fiol MD Department of Anesthesiology, Rutgers - New Jersey Medical School, Newark, NJ

INTRODUCTION

-Aortic Stenosis (AS): most common valvular problem -Prevalence: $>65$ years of age

-Severe AS is associated with a mortality of $43.1 \%^{1}$

-Perioperative mortality rate of $13 \%{ }^{2}$

- Transcatheter aortic valve replacement/implantation (TAVR/TAVI) \& percutaneous aortic balloon dilation (PABD) are options available for patients with severe/critical aortic stenosis who need immediate correction prior to non-cardiac surgeries

-PABD is associated with a mortality of $2-3 \% \&$ post procedure stroke rate of $1-2 \%$.

-TAVR or PABD are bridging strategies for patients that are not candidates for valve replacement.

-Intraoperative management - strict blood pressure and heart rate parameters, within $20 \%$ of baseline values

- Goal is to avoid significant hypotension and tachycardia ${ }^{3}$.

\section{CASE}

86 year old woman with critical aortic stenosis (AV area $\mathbf{0 . 3}$ $\mathrm{cm}^{2}$ ) was scheduled for surgical correction of a left intratrochanteric fracture.

Preoperative echocardiogram showed aortic valve peak pressure of $85 \mathrm{mmHg}$ with a normal ejection fraction and moderate pulmonary hypertension.

Patient initially presented to an outside facility, but was transferred to our hospital given the severity of her condition. Because of the severe aortic stenosis, a balloon valvulopasty was performed to decrease the overall peak gradient to 30 $\mathrm{mmHg}$ prior to surgery.

Cardiology consultation recommended preoperative betablockade along with a short OR time.

Anesthetic plan included pre-induction anxiolysis with midazolam, pre-induction arterial line, and induction with high dose fentanyl.

Maintenance of anesthesia was continued with a remifentanil infusion along with intermittent midazolam boluses. Patient was not a candidate for nitrous oxide given her moderate pulmonary hypertension.

She tolerated the procedure well, without a significant increase in heart rate while maintaining her baseline blood pressure. She was subsequently transferred to the cardiothoracic ICU for overnight monitoring and extubated on POD 1 without any complications.

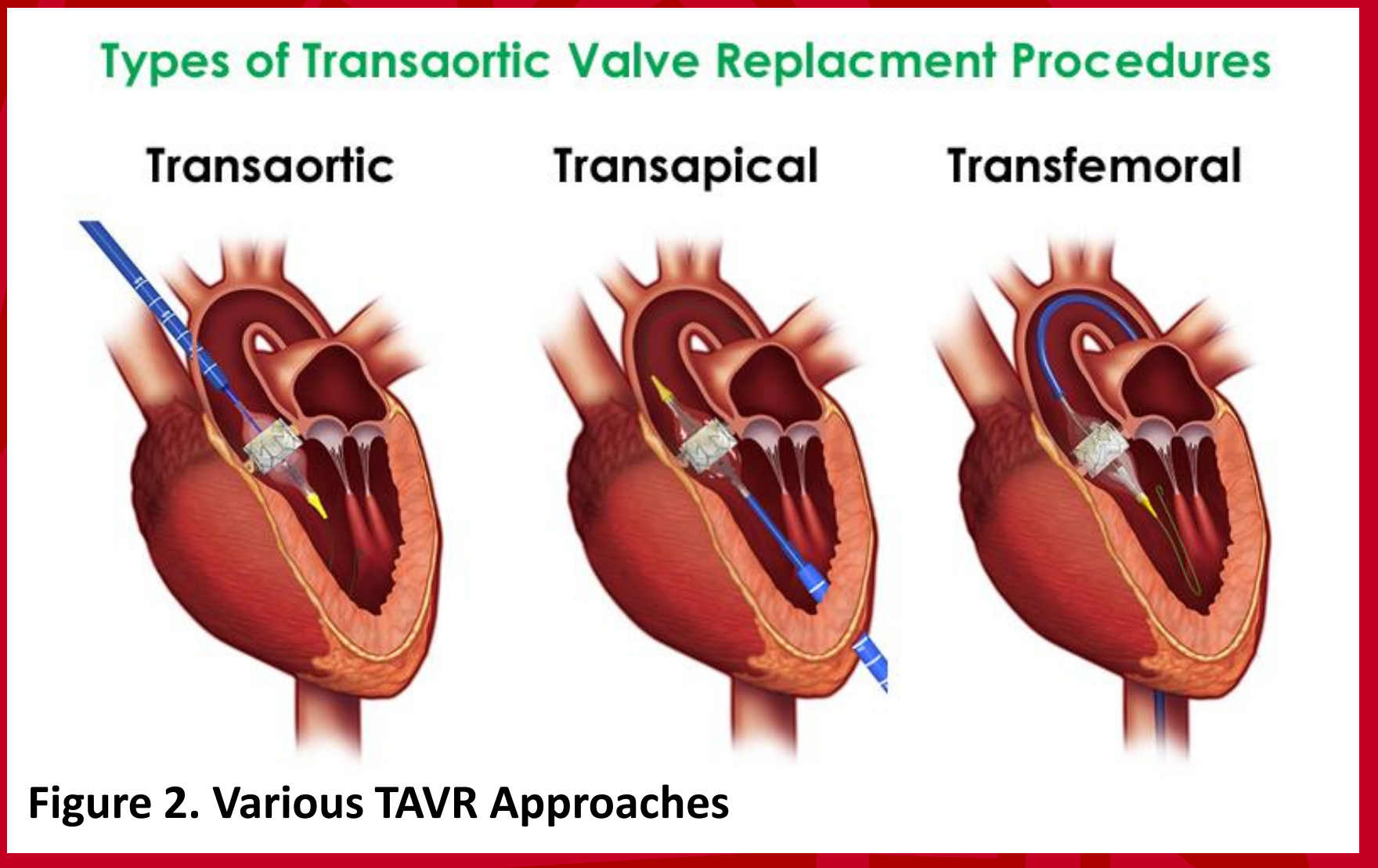

Critical aortic stenosis poses several challenges intraoperatively increasing the morbidity and mortality in patients who are at high risk.

For non-cardiac surgeries, there are several preoperative options that can reduce the post-operatively morbidity and mortality including TAVR/TAVI and PABD. However, these procedures have their own risks and benefits. The permanent solution is a valve replacement. TAVR is an option for those who cannot undergo traditional valve replacement due to high surgical morbidity. Surgical valve replacement still remains the ideal solution to $\mathrm{AS}$, however our patient could not undergo traditional AVR due to poor post op rehab. Both the European and American Heart Association guidelines highlight the importance of appropriate preoperative management of severe aortic stenosis prior to non-cardiac procedures regardless of symptomatic versus asymptomatic valvular pathology.

Our intra-operative approach included TIVA. Although volatile agents could be used, the risk of intra-arterial hypotension with sevoflurane was significant, outweighing any benefits when compared to a total intravenous approach with our high dose opioid induction followed by a remifentanil infusion. There is not enough information in the literature comparing risks and benefits of total intravenous anesthesia versus inhaled anesthesia in cardiac patients undergoing noncardiac procedures ${ }^{3}$. Given that our patient did not have any post-operative complications from her surgery and her balloon valvuloplasty, we would recommend using a total intravenous anesthesia approach intra-operatively.
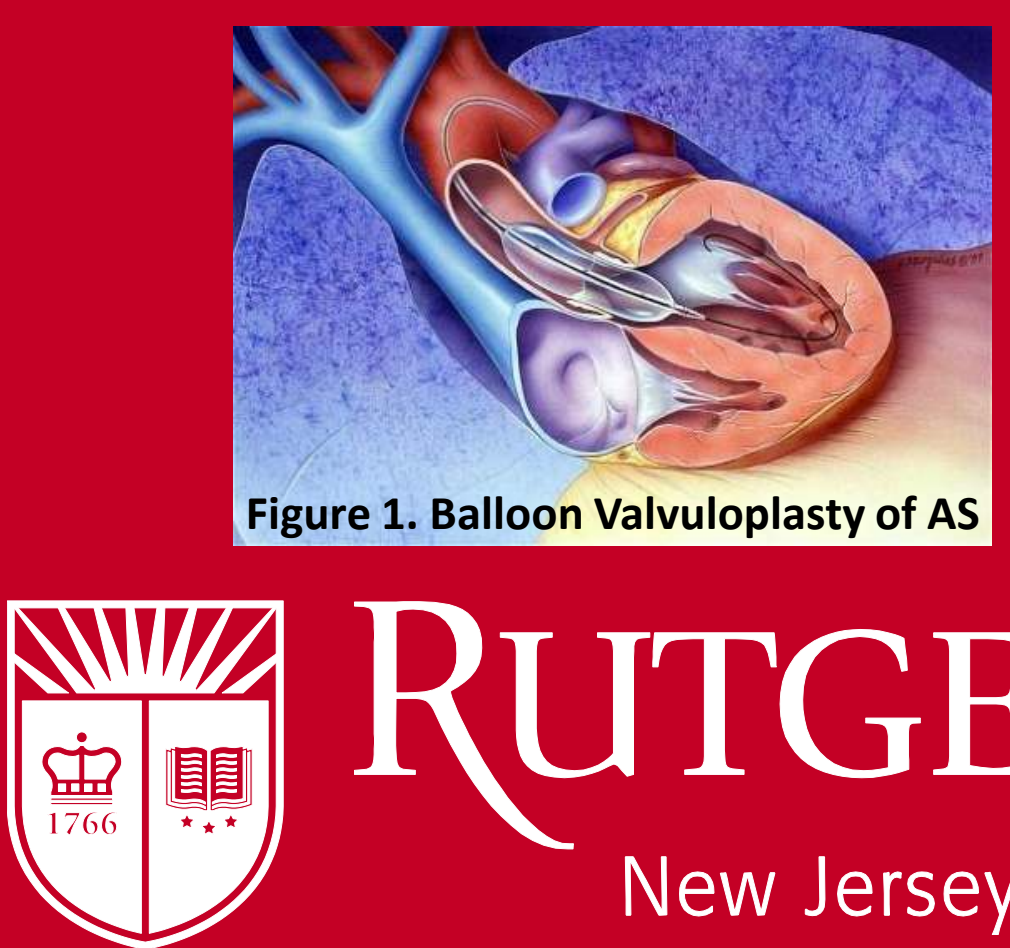

RUTGERS

New Jersey Medical School 